\title{
Control Volume Analysis of Boundary Layer Ingesting Propulsion Systems With or Without Shock Wave Ahead of the Inlet
}

\author{
Hyun Dae $\mathrm{Kim}^{1}$ and James L. Felder ${ }^{2}$ \\ NASA Glenn Research Center, Cleveland, Ohio 44135
}

\begin{abstract}
The performance benefit of boundary layer or wake ingestion on marine and air vehicles has been well documented and explored. In this article, a quasi-one-dimensional boundary layer ingestion (BLI) benefit analysis for subsonic and transonic propulsion systems is performed using a control volume of a ducted propulsion system that ingests the boundary layer developed by the external airframe surface. To illustrate the BLI benefit, a relationship between the amount of BLI and the net thrust is established and analyzed for two propulsor types. One propulsor is an electric fan, and the other is a pure turbojet. These engines can be modeled as a turbofan with an infinite bypass ratio for the electric fan, and with a zero bypass ratio for the pure turbojet. The analysis considers two flow processes: a boundary layer being ingested by an aircraft inlet and a shock wave sitting in front of the inlet. Though the two processes are completely unrelated, both represent a loss of total pressure and velocity. In real applications, it is possible to have both processes occurring in front of the inlet of a transonic vehicle. Preliminary analysis indicates that the electrically driven propulsion system benefits most from the boundary layer ingestion and the presence of transonic shock waves, whereas the benefit for the turbojet engine is near zero or negative depending on the amount of total temperature rise across the engine.
\end{abstract}

\section{Nomenclature}

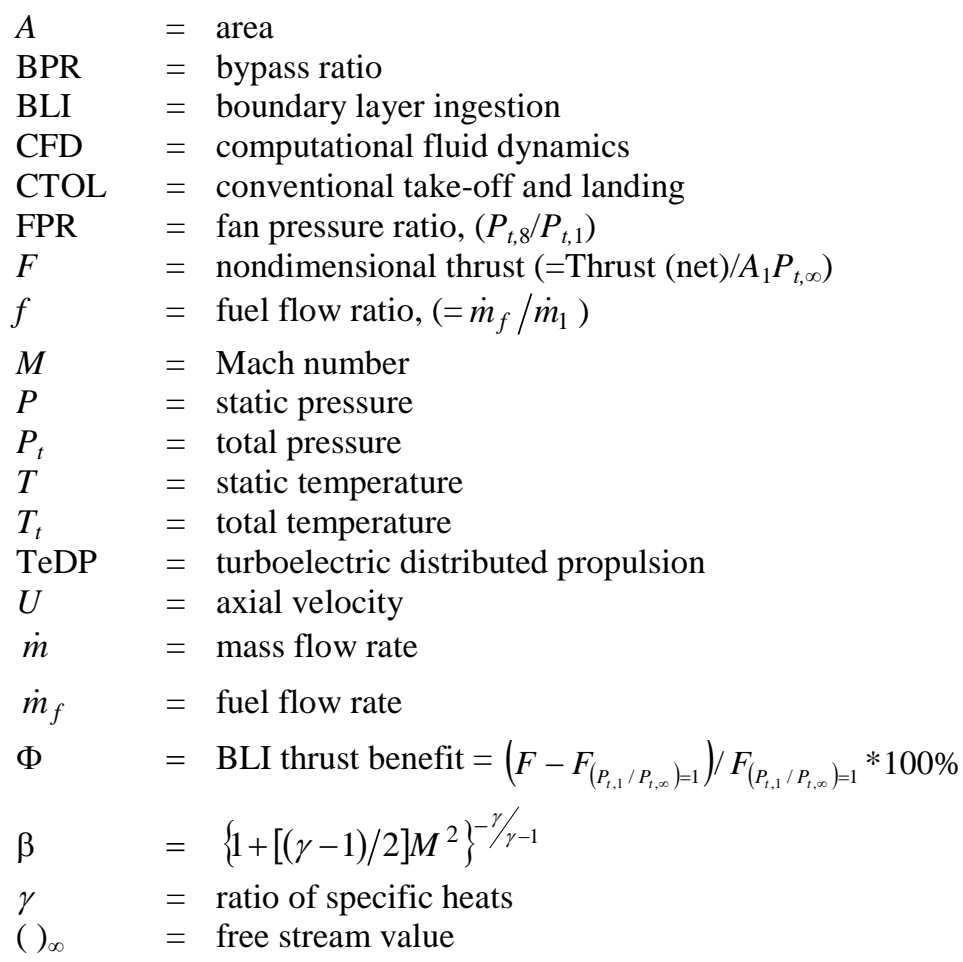

\footnotetext{
${ }^{1}$ Aerospace Engineer, Aeropropulsion Division, 21000 Brookpark Road, Mail Stop 5-11, Senior Member

${ }^{2}$ Aerospace Engineer, Systems Engineering and Analysis Division, 21000 Brookpark Road, Mail Stop 86-4, Member
} 

()$_{s} \quad=$ value after shock wave
()$_{0} \quad=$ inlet ambient value
()$_{1} \quad=$ inlet throat value
()$_{8} \quad=$ nozzle exit value
()$_{8}, \quad=$ nozzle exit ambient value

\section{Introduction}

$\mathrm{W}$

ith increased interest in electric propulsor concepts such as turboelectric distributed propulsion ${ }^{1-3}$ (TeDP), the boundary layer ingestion (BLI) benefit analysis becomes especially important. The vehicle takes credit for large amount of fuel burn reduction as a result of BLI. A concept currently being analyzed at NASA is a conventional take-off and landing (CTOL) vehicle designated as N3-X shown in Fig.1. The vehicle utilizes distributed electrically driven low-pressure ratio fans with power provided by two remote electric generators driven by conventional turbofan or turboshaft core engines. The use of electrical power transmission allows a high degree of flexibility in positioning the turbogenerators and propulsor modules. In the aircraft configuration examined, the turbogenerators are located at the wing tips, where the turbogenerators would see undisturbed freestream condition. The fan modules are positioned in a continuous fan nacelle across the rear fuselage where they ingest the upper airframe surface boundary layer flow and thereby reduce the thrust required by the vehicle. Although large transport vehicle concepts such as N3-X may benefit from the highly integrated airframe and propulsion systems, they also presents challenges that are not traditionally considered in large transport design. By embedding the propulsion system within the airframe, the fan inlet flow field at the inlet lip station does not experience undisturbed free stream flow as in a conventional "tube and wing" transport but rather sees upper airframe surface boundary layer flow plus the airframe inviscid flow, which could be quite different from the free stream condition.
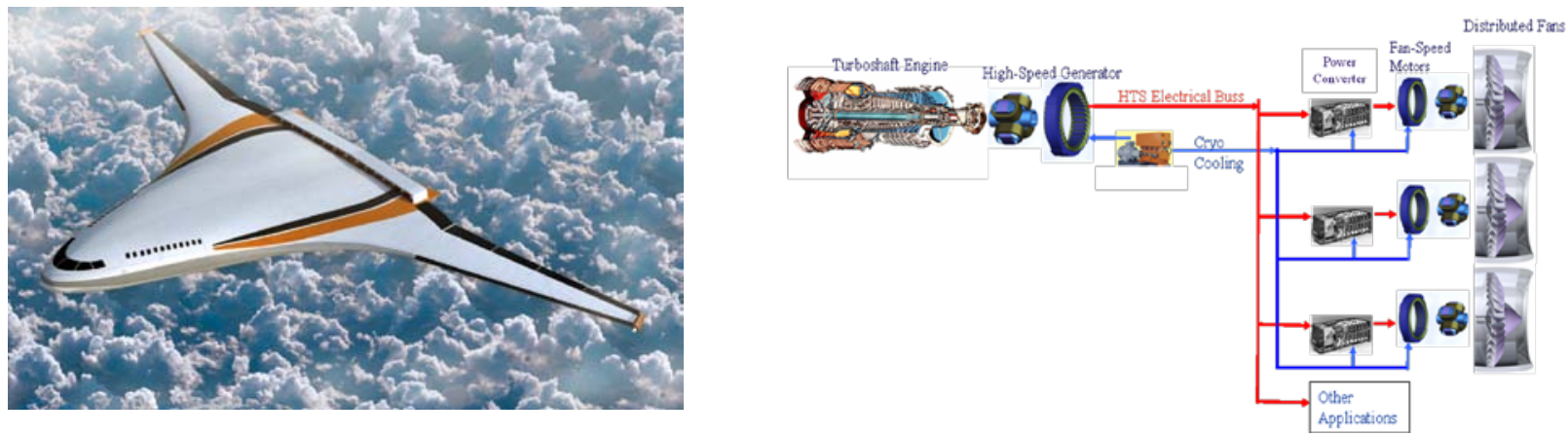

Figure 1. Turboelectric Distributed Propulsion (TeDP) N3-X vehicle and a schematic of its propulsion system.

Recent papers have addressed the BLI benefits using theoretical approaches, computational fluid dynamics (CFD), and experimental analyses. ${ }^{4-7}$ These papers clearly show that there is a net benefit, in terms of thrust needed and power required to propel an aircraft, if the inlet ingests a portion of the aircraft boundary layer flow and fills in the wake with thrust stream. In this paper, the coupling effect of BLI and total temperature or pressure rise through a ducted propulsion system is investigated. This analysis is especially useful to quantify the benefit when the airframe and propulsion system are highly integrated such as multifan distributed propulsion vehicles. As shown in Fig. 1, the multiple propulsors are linearly arranged in the spanwise direction in order to ingest and re-accelerate a large fraction of the boundary layer flow occurring on the top of the airframe surfaces.

For the case where the lifting device travels at transonic speed, a shock wave may be present ahead of the embedded inlet, as shown in Fig. 2. The net BLI benefit of boundary layer flow with shock wave interactions has not been previously analyzed. This paper provides a first look at this problem by using a control volume analysis. Shock waves and boundary layer flows are completely unrelated processes, yet both represent a loss of total pressure and velocity. In real applications, it is possible to have both processes occurring in front of inlet for a transonic vehicle. 


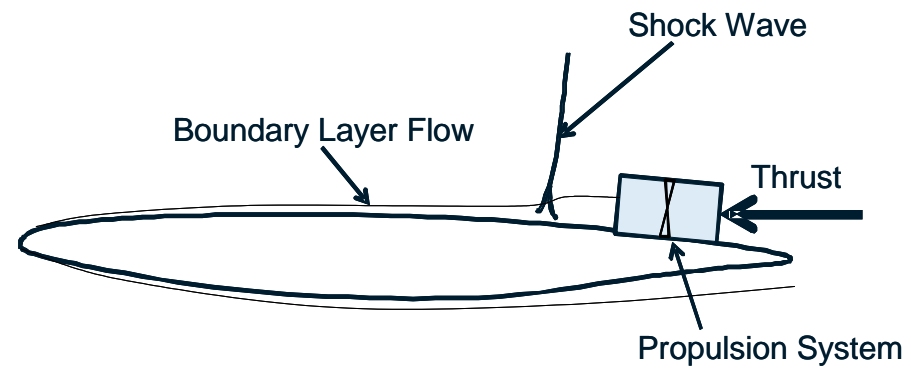

Figure 2. Schematic of BLI propulsion system with transonic shock waves on an airfoil.

\section{Analysis and Results}

In order to simplify the analysis and to illustrate the impact of BLI and shock waves in front of an inlet on the propulsion system performance, several assumptions are made. First, a quasi-one-dimensional thrust analysis is performed using a control volume to assess the impacts on propulsion system. Although the flow conditions from the airframe to the nacelle surface vary because of the boundary layer flow and local inviscid flow, the average flow properties are used at the inlet entrance and nozzle exit stations. Second, only a subsonic converging choked nozzle is considered. This condition means that the nozzle exit Mach number is unity because the nozzle exit static pressure is equal to the local nozzle exit ambient static pressure.

In this study, the total pressure loss due to boundary layer flow and shock wave is varied to obtain net thrust (gross thrust minus ram drag) in a nondimensional form. Although the propulsion system inside the control volume could be any propulsor type (turbojet, turbofan, electric fan, cross-flow-fan, ramjet, etc.), only two extreme cases are considered. An electric fan propulsor can be considered as a turbofan with an infinite fan bypass ratio (BPR) because there is no engine core flow. A turbojet propulsor can be considered as a turbofan with the BPR equal to zero because there is no fan directly attached to the engine core. Applying these two limiting cases of propulsor types, the analysis of conventional turbofan with separate nozzle could be easily studied by separating the flow stream into fan flow and engine core flow and then combining the results at the end.

\section{A. Analysis for Propulsion System With Boundary Layer Integration}

The control volume in Fig. 3 is used to estimate net thrust for the propulsion system. A similar approach is taken for the cases with a transonic shock wave and will be described in a later section. Assuming quasi-one-dimensional flow, the net thrust for the propulsion system can be written as follows:

$$
\text { Thrust (net) }=\left(\dot{m}_{1}+\dot{m}_{f}\right) U_{8}-\dot{m}_{1} U_{1}+\left(P_{8}-P_{8^{\prime}}\right) A_{8}-\left(P_{1}-P_{0}\right) A_{1}
$$

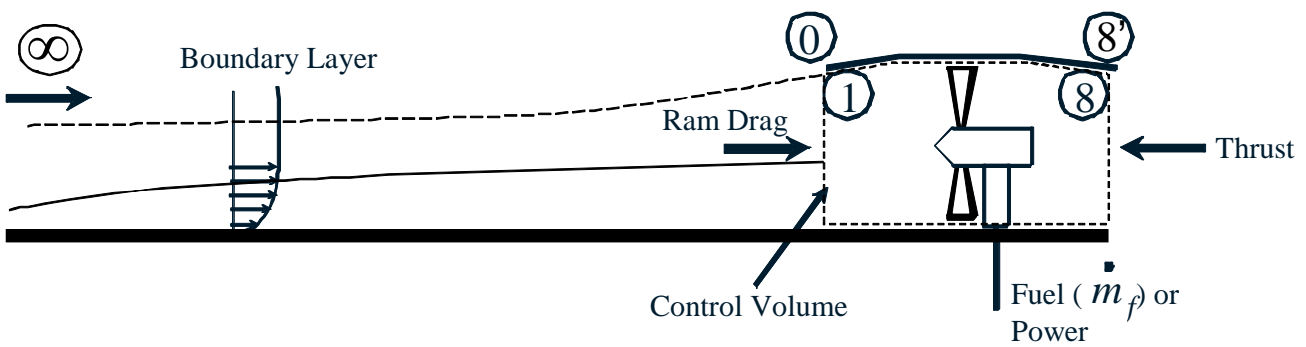

Figure 3. Quasi-one-dimensional control volume of propulsion system ingesting airframe boundary layer flow.

For subsonic flight conditions and a perfectly expanded nozzle, nozzle exit static pressure $P_{8}$ is equal to nozzle exit ambient pressure $P_{8}$. Then, 


$$
\text { Thrust (net) }=\dot{m}_{1}\left[(1+f) U_{8}-U_{1}\right]-\left(P_{1}-P_{0}\right) A_{1} \quad \text { where } f=\frac{\dot{m}_{f}}{\dot{m}_{1}}
$$

Introducing the Mach number $M$ and compressible flow relations for the pressure, and nondimensionalizing the thrust by the product of the inlet area $\left(A_{1}\right)$ and free-stream total pressure $\left(P_{t, \infty}\right)$, the net thrust equation becomes

$$
F=\frac{\text { Thrust (net) }}{A_{1} P_{t, \infty}}=\left[\gamma(1+f) M_{1} M_{8} \beta_{1} \sqrt{\frac{T_{t, 8}}{T_{t, 1}}} \sqrt{\frac{\left(1+\frac{\gamma-1}{2} M_{1}^{2}\right)}{\left(1+\frac{\gamma-1}{2} M_{8}^{2}\right)}}\right] \frac{P_{t, 1}}{P_{t, \infty}}-\left[\beta_{1}\left(\gamma M_{1}^{2}+1\right)\right] \frac{P_{t, 1}}{P_{t, \infty}}+\beta_{0}
$$

where

$$
\begin{aligned}
& \beta=\left(1+\frac{\gamma-1}{2} M^{2}\right)^{-\frac{\gamma}{\gamma-1}} \\
& P=P_{t}\left(1+\frac{\gamma-1}{2} M^{2}\right)^{-\frac{\gamma}{\gamma-1}} \\
& T=T_{t}\left(1+\frac{\gamma-1}{2} M^{2}\right)^{-1}
\end{aligned}
$$

In the above equation, $P_{t, 0}$ is replaced by $P_{t, \infty}$ because the value of total pressure for inviscid flow in front of the inlet along the axial direction is constant. Although the Eq. (3) is nondimensionalized by the product of the inlet throat area and free-stream total pressure, it could also be based on the nozzle exit area using the conservation of mass equation (i.e., $A_{8}$ is the product of $A_{1}$ and the function of $P_{t, 8} / P_{t, 1}, T_{t, 8} / T_{t, 1}, M_{1}$, and $M_{8}$ ). This approach was not taken because of the complexity introduced by variable nozzle area as required by some conventional propulsion systems. However, the use of $A_{1}$ in Eq. (3) does not imply that a variable area inlet cannot be analyzed. A configuration like N3-X may adopt a variable cowl lip for enhanced aerodynamic interaction between the airframe and the external nacelle. The two-dimensional inlet lip structure could be easily adjusted to the incoming flow for the mass flow required by the propulsors without suffering from external flow separation around the inlet lip.

In order to illustrate the benefit associated with BLI with or without a transonic shock in front of the inlet, the following definition for the thrust benefit parameter is used:

$$
\Phi=\left[\frac{F-F_{\left(P_{t}, 1 / R, \infty\right)=1}}{F_{\left(P_{t, 1} / P_{, \infty}\right)=1}}\right] \cdot 100 \%
$$

The thrust benefit $(\Phi)$ is the percentage in increased net thrust of BLI propulsion system compared with the identical propulsion system in free stream without any total pressure loss ahead of inlet.

Case 1. Boundary layer ingestion benefit using electric fan as propulsor

For this case the fan propulsors are powered by electric motors to provide thrust required by the aircraft. The N3-X propulsion system uses multiple distributed electric fans to maximize propulsive efficiency while also maximizing thermal efficiency of the turboelectric generator. The propulsors are mechanically decoupled from the turbine engine core by connecting them using electric transmission. Although there are inefficiencies related to the axial fan and losses through the internal duct from the inlet to nozzle, an ideal condition for both the fan and the duct is applied to simplify and to illustrate the electric fan characteristics in the presence of BLI. In addition, in order to use a commonly known industry fan design variable, the total temperature ratio $\left(T_{t, 8} / T_{t, 1}\right)$ in Eq. (3) is converted to the fan pressure ratio (FPR $=P_{t, 8} / P_{t, 1}$ ) by assuming the following isentropic relationship exists between the inlet lip and nozzle exit stations: 


$$
\frac{T_{t, 8}}{T_{t, 1}}={\frac{P_{t, 8}}{P_{t, 1}}}^{\left(\frac{\gamma-1}{\gamma}\right)}=(\mathrm{FPR})^{(\gamma-1 / \gamma)}
$$

Also, since the electric fan requires no fuel added in the control volume, the fuel flow ratio is zero $(f=0)$.

With the assumption of $M_{8}=1$ for a choked nozzle, Eq. (3) then becomes

$$
F=\frac{\text { Thrust (net) }}{A_{1} P_{t, \infty}}=\left[\gamma M_{1} \beta_{1}(F P R)^{\frac{\gamma-1}{2 \gamma}} \sqrt{\frac{\left(1+\frac{\gamma-1}{2} M_{1}^{2}\right)}{\left(1+\frac{\gamma-1}{2}\right)}}\right] \frac{P_{t, 1}}{P_{t, \infty}}-\left[\beta_{1}\left(\gamma M_{1}^{2}+1\right)\right] \frac{P_{t, 1}}{P_{t, \infty}}+\beta_{0}
$$

This equation relates nondimensional net thrust to the total pressure recovery at inlet throat station 1 , given the fan design variable FPR, the flow properties at free-stream and around the inlet, and the Mach number at the inlet throat.

In order to illustrate the benefit of BLI, a sample case is considered where the area of the streamtube entering the inlet is the same as the one at free stream. In this case, there is no flow diffusion or contraction entering the inlet (i.e., $\left.A_{\infty}=A_{1}\right)$ but the average Mach number at inlet throat $\left(M_{1}\right)$ is varied with the amount of BLI or total pressure recovery, $P_{t, 1} / P_{t, \infty}$. For other cases, the inlet ambient Mach number external to inlet capture or throat area $\left(M_{0}\right)$ must be determined in some other way so it can be used directly in Eq. (6).

Fig. 4(a) shows a plot of nondimensional thrust benefit as a function of $P_{t, 1} / P_{t, \infty}$, or varying degrees of BLI, for five different FPRs at $M_{0}=M_{\infty}=0.8$. In Fig. 4(b) a similar plot shows BLI benefit against various FPRs for four different amount of total pressure recovery at $M_{0}=M_{\infty}=0.8$. From the figure it can be seen that the net thrust benefit increases as the inlet ingests more boundary layer flow (i.e., lower $P_{t, 1} / P_{t, \infty}$ ). This result is consistent with the results from others in References 4 to 7. Another important result from this figure is that the BLI benefit increases with lower FPR and lower total pressure recovery. Although Fig 4(a) and (b) seem to indicate that it is always preferable to use lower FPR for the fan, the lower FPR means that more mass flow is needed to provide a desired level of thrust. The increased mass flow can be obtained either from more boundary layer flow in the spanwise direction, or more inviscid flow in the vertical direction. If the nacelle span width is fixed as it is for N3-X, more inviscid flow must be ingested by the inlet in order to provide a desired thrust level. The increased inviscid flow produces higher pressure recovery, which in turn may lower the BLI benefit.

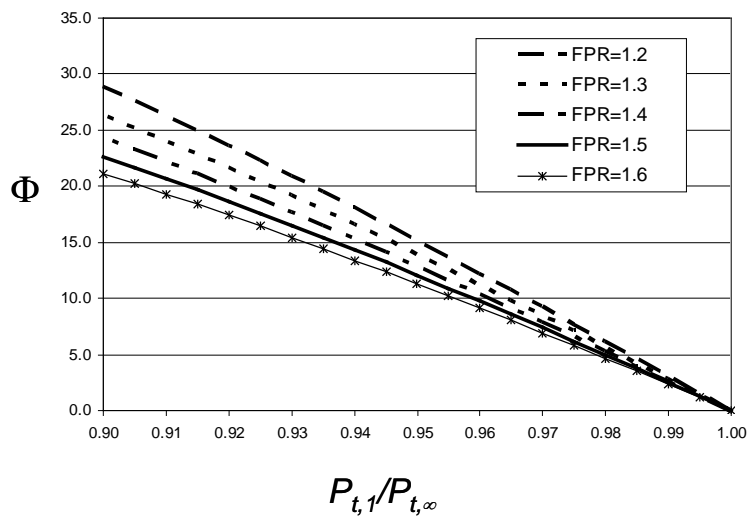

(a)

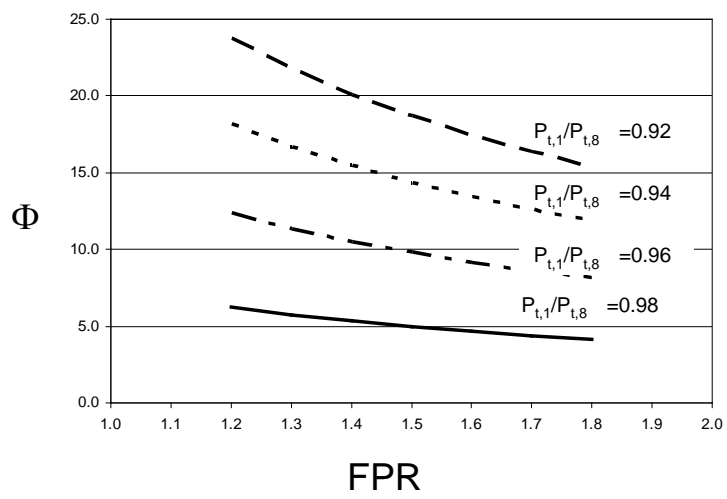

(b)

Figure 4. (a) Amount of BLI $\left(P_{t, 1} / P_{t, \infty}\right)$ vs. nondimensional thrust benefit in percentage, $\Phi$, and (b) fan pressure ratio (FPR) vs. $\Phi$ for ducted electric fan system at $M_{\infty}=M_{0}=0.8$. 
Case 2. Boundary layer ingestion benefits using a gas turbine engine with fan bypass ratio equal to zero.

For this case the thrust is provided only by high-pressure combustion air. Two examples of this type of propulsor are a pure turbojet engine with bypass ratio of zero or a core engine without directly attached fans. The later example occurs when the core engine drives remotely located multiple fans by mechanical, fluidic, or electric means. For this case, $T_{\mathrm{t}, 8}$ is the total temperature exiting the nozzle and is a function of the turbomachinery performance and the amount of combustion in the combustor.

Figure 5 shows the results of sample calculations using Eq. (3). It shows a plot of nondimensional thrust benefit variation as a function of boundary layer ingestion amount at five different total temperature ratios $\left(T_{t, 8} / T_{t, 1}\right)$. In this figure, a nominal fuel flow ratio $(f)$ of 0.029, free-stream Mach number of 0.8 , and inlet ambient Mach number of 0.8 were used to investigate the effect of BLI on thrust benefit. As before, $M_{1}$ is varied with different amount of BLI to maintain constant streamtube area in front of the inlet. As the engine ingests more boundary layer, the total pressure ratio decreases. From the figure, it can be seen that the net thrust benefit is near zero or negative depending on the total temperature ratio. If it is assumed that the total temperature ratio across a current conventional turbofan engine core is nominally 2.6, then this engine or engine core produces negative net thrust benefit with any amount of BLI. Furthermore, as engine technology improves, the total temperature ratio across the engine tends to go higher and possibly may extend beyond 5. If this becomes the case for future engine, then Fig. 5 indicates more negative net thrust benefit is obtained as the engine ingests more boundary layer flow. Although this is a sample case, this result is significantly different from the case with electric fan.

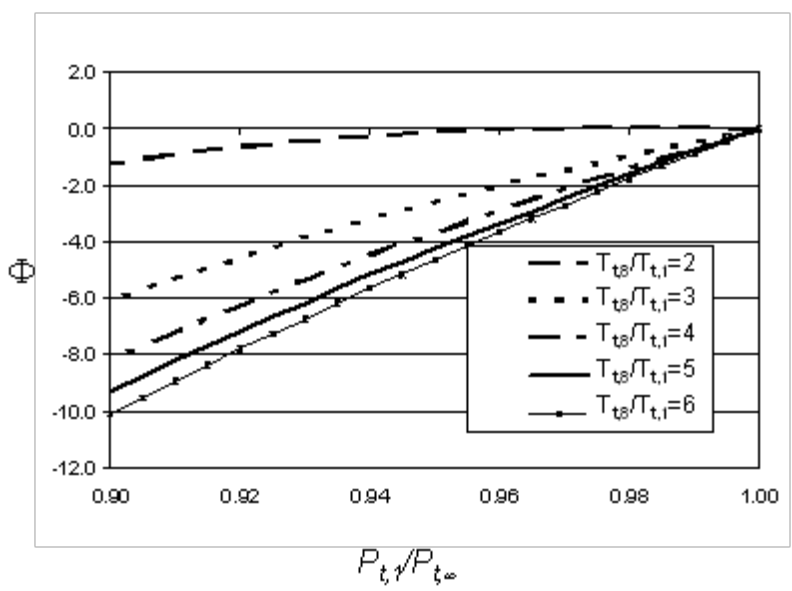

Figure 5. Amount of BLI $\left(P_{t, 1} / P_{t, \infty}\right)$ vs. nondimensional thrust benefit in percentage, $\Phi$, for turbojet-like engine without fans at various $T_{t, 8} / T_{t, 1} \cdot\left(f=0.029, M_{\infty}=M_{0}=0.8\right)$.

For the next two test cases, we shall consider an aircraft travelling at transonic speeds for which a shock wave is present upstream of the imbedded propulsion system.

\section{B. Analysis for Propulsion System With Shock Wave Ahead of Unlet in Inviscid Flow}

For this analysis, a standing shock wave is assumed to be located upstream of the propulsion system, and the streamtube entering the inlet could be enlarged or decreased depending on engine flow demand. An analysis similar to the BLI analysis is presented using the control volume in Fig. 6 to estimate net thrust for the propulsion system. Assuming inviscid quasi-one-dimensional flow, the net thrust for the propulsion system could be written as follows:

$$
\text { Thrust (net) }=\left(\dot{m}_{1}+\dot{m}_{f}\right) U_{8}-\dot{m}_{1} U_{1}+\left(P_{8}-P_{8^{\prime}}\right) A_{8}-\left(P_{1}-P_{0}\right) A_{1}
$$




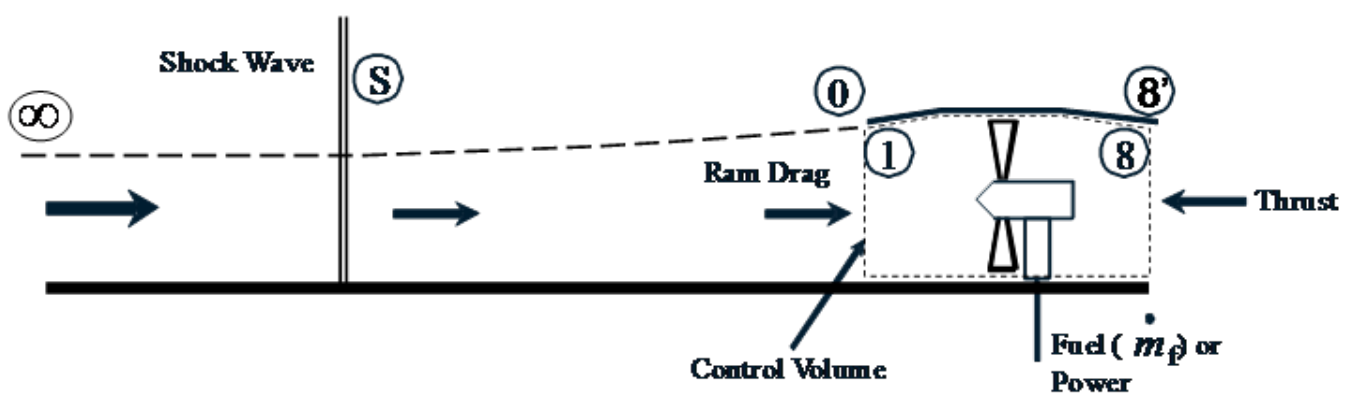

Figure 6. Quasi-one-dimensional control volume of propulsion system in inviscid flow with a shock wave in front of inlet.

After algebra, the nondimensional thrust $F_{s}$ based on inlet area and total pressure after the shock wave becomes

$$
F_{S}=\frac{\text { Thrust (net) }}{A_{1} P_{t, s}}=\left\{\gamma(1+f) M_{1} M_{8} \beta_{1} \sqrt{\frac{T_{t, 8}}{T_{t, 1}}} \sqrt{\frac{\left(1+\frac{\gamma-1}{2} M_{1}^{2}\right)}{\left(1+\frac{\gamma-1}{2} M_{8}^{2}\right)}}-\beta_{1}\left(\gamma M_{1}^{2}+1\right)+\beta_{0}\right\}
$$

where $f$ and $\beta$ are defined earlier in Eq. (3).

In order to express the Eq. (8) in terms of nondimensional thrust $F$ based on free-stream total pressure $P_{t, \infty}$ rather than $P_{t, s}$, Eq. (8) is multiplied by $P_{t, s} / P_{t, \infty}$ on both sides of equation. Then, the resulting equation becomes

$$
F=\frac{\text { Thrust(net) }}{A_{1} P_{t, \infty}}=\left\{\gamma(1+f) M_{1} M_{8} \beta_{1} \sqrt{\frac{T_{t, 8}}{T_{t, 1}}} \sqrt{\frac{\left(1+\frac{\gamma-1}{2} M_{1}^{2}\right)}{\left(1+\frac{\gamma-1}{2} M_{8}^{2}\right)}}-\beta_{1}\left(\gamma M_{1}^{2}+1\right)+\beta_{0}\right\} \frac{P_{t, s}}{P_{t, \infty}}
$$

One word of caution is that, although the above equation looks to have linear relationship between $F$ and total pressure recovery $P_{t, s} / P_{t, \infty}$ across shock wave, the inlet ambient Mach number after the shock, $M_{0}$ in $\beta_{0}$, is directly related to $P_{t, s} / P_{t, \infty}$ and therefore, one should not assume that the above equation is linear.

Case 3. Effect of shock wave ahead of propulsion system in inviscid transonic flow

This case is similar to the case in which a transonic airfoil in inviscid flow experiences supersonic speed over part of the airfoil. A shock wave is formed on the airfoil to bring the flow back to subsonic free stream conditions. The shock wave then sits in front of the imbedded propulsion system inlet. Applying Eq. (9) directly to a transonic airfoil case must be done carefully because the flow ahead of the shock is not the free stream flow but rather it is a supersonic flow resulting from flow acceleration above the airfoil.

In order to illustrate the transonic shock effect on the propulsion system, a sample case with $M_{s}=M_{0}=M_{1}=0.8$ is considered such that there is no flow diffusion or contraction for the flow going into the inlet. For this case, the Mach number ahead of the shock is 1.273 , and the total pressure recovery across the shock is 0.984 . Figure 7 shows the nondimensional thrust obtained from Eq. (9) for various electric fan FPRs compared to the cases without shock. For the case with the shock, the flow is assumed to start at Mach 0.8 at free stream, accelerate to Mach 1.273 because of a transonic pressure gradient, and decelerate to Mach 0.8 again through the normal shock wave. For transonic airfoils, this acceleration to Mach 1.273 is unlikely but was used to demonstrate the effect by the shock wave on the propulsion system. The result was a reduction of $1.6 \%$ in thrust benefit due to the shock wave for FPRs 
between 1.2 and 1.8 for electric fan. A similar 1.6\% reduction was also obtained when a turbojet-like propulsor was studied by varying the values of $T_{t, 8} / T_{t, 1}$ from 2 to 6 . The reduction of $1.6 \%$ in benefit is directly related to the decrease in total pressure recovery across the shock wave for the case of $M_{s}=M_{0}=M_{1}=0.8$. The benefit amount may differ from the percentage decrease in total pressure recovery if the inlet throat Mach number differs from the Mach number downstream of the shock. The change in Mach number occurs if there is diffusion or contraction behind the shock but ahead of the inlet.

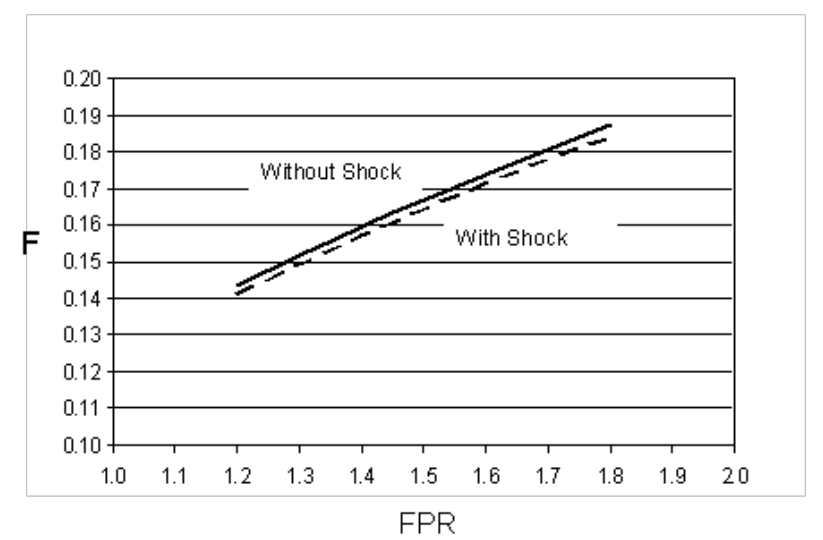

Figure 7. Mach 1.273 transonic shock effect on nondimensional thrust, $F$, by fan pressure ratio (FPR) for electric fan at $M_{\infty}=M_{0}=0.8$.

\section{Analysis for Propulsion System With Shock Wave Ahead of Inlet in Viscous Flow}

An analysis similar to the above two analyses is conducted for the case of boundary layer ingestion with a transonic shock wave in front of the inlet. As shown in Fig. 8, a standing shock wave is assumed to be located upstream of the propulsion system, and the inlet is ingesting the boundary layer flow generated by the airframe surface. Assuming quasi-one-dimensional viscous flow and a simple normal shock and boundary layer flow rather than a complex boundary layer shock wave interaction involving possible flow recirculation underneath the lambda shock, a similar analysis results in the following equation:

$$
\begin{aligned}
& F=\frac{\text { Thrust (net) }}{A_{1} P_{t, \infty}}=\left\{\gamma(1+f) M_{1} M_{8} \beta_{1} \sqrt{\frac{T_{t, 8}}{T_{t, 1}}} \sqrt{\frac{\left(1+\frac{\gamma-1}{2} M_{1}^{2}\right)}{\left(1+\frac{\gamma-1}{2} M_{8}^{2}\right)}}\right\} \frac{P_{t, 1}}{P_{t, \infty}} \\
& -\left[\beta_{1}\left(\gamma M_{1}^{2}+1\right)\right] \frac{P_{t, 1}}{P_{t, \infty}}+\beta_{0} \frac{P_{t, 0}}{P_{t, \infty}}
\end{aligned}
$$

It is noted that the only difference between Eq. (10) and Eq. (3) is the additional term of $P_{t, 0} / P_{t, \infty}$ after $\beta_{0}$ in Eq. (10). Since the total pressure at the inlet throat station, $P_{t, 1}$, is due to both boundary layer ingestion and the shock wave, and assuming only a simple normal shock wave exists above the airframe, then Eq. (10) then can be expressed as

$$
F=\frac{\text { Thrust (net) }}{A_{1} P_{t, \infty}}=\left\{\gamma(1+f) M_{1} M_{8} \beta_{1} \sqrt{\frac{T_{t, 8}}{T_{t, 1}}} \sqrt{\frac{\left(1+\frac{\gamma-1}{2} M_{1}^{2}\right)}{\left(1+\frac{\gamma-1}{2} M_{8}^{2}\right)}}-\beta_{1}\left(\gamma M_{1}^{2}+1\right)\right\}\left(\left.\left.\frac{P_{t, 1}}{P_{t, \infty}}\right|_{B L I} X \frac{P_{t, 1}}{P_{t, \infty}}\right|_{\text {Shock }}\right)+\beta_{0} \frac{P_{t, s}}{P_{t, \infty}}
$$


where $P_{t, 0}$ is substituted by $P_{t, s}$ since they are equal behind the shock.

Further simplifying the above equation, it becomes

$$
F=\frac{\text { Thrust (net) }}{A_{1} P_{t, \infty}}=\left\{\left.\left[\gamma(1+f) M_{1} M_{8} \beta_{1} \sqrt{\frac{T_{t, 8}}{T_{t, 1}}} \sqrt{\frac{\left(1+\frac{\gamma-1}{2} M_{1}^{2}\right)}{\left(1+\frac{\gamma-1}{2} M_{8}^{2}\right)}}-\beta_{1}\left(\gamma M_{1}^{2}+1\right)\right] \frac{P_{t, 1}}{P_{t, \infty}}\right|_{\text {BLI }}+\beta_{0}\right\} \frac{P_{t, s}}{P_{t, \infty}}
$$

where $f$ and $\beta$ are defined earlier in Eq. (3).

Applying Eq. (12) to BLI only without a shock wave, the value of $P_{t, s} / P_{t, \infty}$ becomes unity, and the equation reverts back to Eq. (3). Similarly, applying the Eq. (12) to the case with only a shock wave, the $\mathrm{P}_{\mathrm{t} 1} / \mathrm{P}_{\mathrm{t} o}$ due to BLI becomes unity and the equation reverts back to Eq. (9).

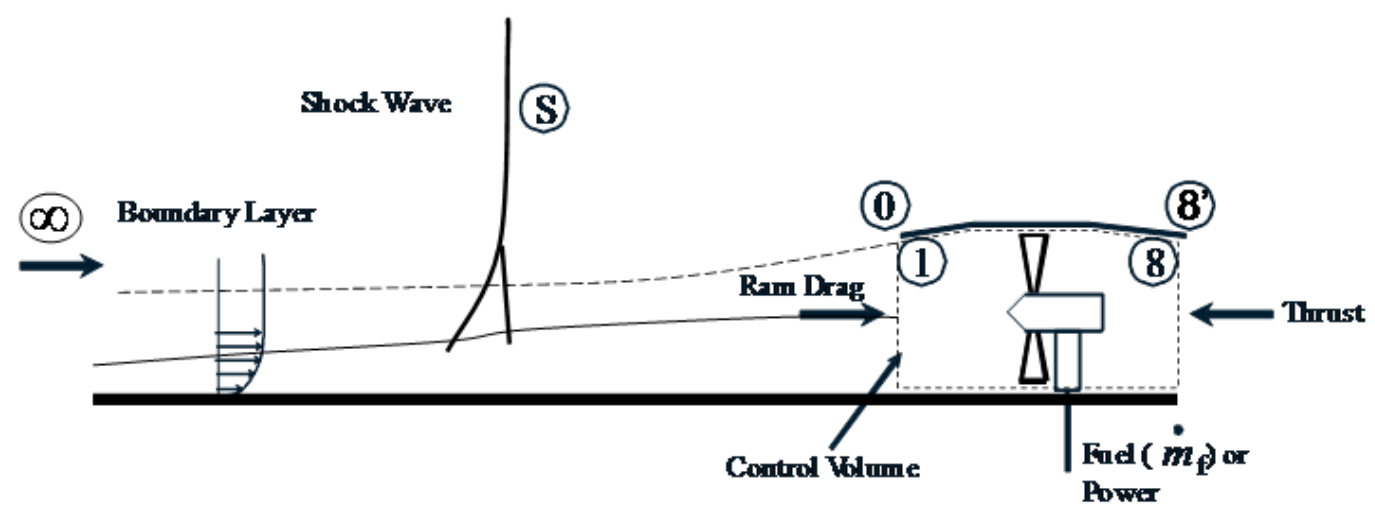

Figure 8. Quasi-one-dimensional control volume of propulsion system ingesting airframe boundary layer flow with transonic shock waves in front of inlet.

Case 4. Effect of shock waves ahead of BLI inlet on transonic aircraft

The shock wave effect on the BLI inlet is directly related to the total pressure recovery drop across the shock and the Mach number after the shock. Equation (12) shows that, for an example case of $M_{\infty}=M_{1}=0.8$ and the total pressure recovery loss of $1.6 \%$ across the shock wave, the reduction in BLI benefit due to the shock is the same amount of $1.6 \%$ reduction from the benefit obtained from the boundary layer ingestion alone. However, since there is a close relationship between the Mach number after the shock wave and the total pressure recovery loss across the shock, any variation of the Mach number after the shock from the inlet throat Mach number will result in different amounts of loss in the BLI benefit. In order to estimate more realistic effect of BLI and shock waves on the propulsion system, it is critical to determine the inlet ambient flow condition before Eq. (12) is applied.

\section{Discussion}

For the electric fan propulsion system, the quasi-one-dimensional analysis indicates that ingesting the boundary layer flow from the airframe surface produces significant thrust benefit for the fan pressure ratio examined. It is also shown from Fig. 4 that the benefit could be increased if a lower FPR fan is used and ingests more boundary layer flow. However, for a fixed amount of boundary layer flow and inlet width, the use of lower FPR means that the mass flow rate must be increased for the same vehicle required thrust, and therefore inlet height must be increased to accommodate the required inlet mass flow. This in turn increases the average total pressure recovery at the inlet throat station and points to lower net thrust benefit. 
The BLI benefit associated with turbojet engine or similar types of propulsors such as the engine core without a directly attached fan seems to be almost negligible or negative depending on the total temperature rise across the engine, and therefore it is not desirable to ingest boundary layer flow from the airframe for the turbojet-like engines. In addition, any significant flow distortion associated with the boundary layer flow entering the inlet may propagate through the engine compressor and will lower the compressor efficiency and hence the thermodynamic efficiency of the engine.

Combining both cases of electric fan and turbojet-like engine cases could enable the analysis of conventional turbofan with BLI inlet. Depending on the amount of the boundary layer flow going into the fan and the engine core, the effect on the net thrust benefit may differ. For example, if $100 \%$ of boundary layer flow is diverted to the fan flow, a similar approach to electric fan analysis could be done to estimate fan thrust benefit. Since no boundary layer flow is entering the engine core, a turbojet approach could be used to estimate the benefit related to engine core thrust. Then, these two analyses could be combined into one by considering the engine bypass ratio and thrusts from the fan and the engine core.

A shock wave above the wing is highly possible for transonic aircraft. The shock wave boundary layer interaction is a very complicated phenomenon. Depending on how much laminar flow is extended on the top of airfoil or airframe, the shock wave will most likely trigger flow transition to turbulent flow. In addition, the pressure gradient on the airfoil surface will invariably affect the boundary layer buildup and interact with the shock wave, possibly establishing flow recirculation underneath the lambda shock. A simplified approach to this complex problem was taken for this study by assuming only a normal shock wave on the top of airframe surface and ignoring the complex flow interaction between the shock wave and boundary layer flow. Some example shock wave cases examined are very specific in terms of in-flow Mach number and inlet throat Mach number. The intent was to illustrate the effect of the shock on the propulsion system. In order to apply the equations used in the above analysis to more general flow field, an estimation of the inlet ambient Mach number and the flow condition after the shock wave must be obtained.

Finally, the current analysis is still being refined, and the equations used in the above analysis are currently limited to propulsion system at subsonic speed with perfectly expanded choked nozzle. In the future, the analysis may include the propulsion system with unchoked nozzle and even a supersonic propulsion system. Based on the current analysis, the electric fan propulsion system seems to show promising results for subsonic aircraft in reducing aircraft fuel burn through the use of lower pressure ratio fans and the airframe boundary layer ingestion.

\section{Conclusion}

A quasi-one-dimensional boundary layer ingestion benefit analysis for subsonic and transonic propulsion system is performed using a control volume of a ducted propulsion system that ingests the boundary layer flow developed by the external airframe surface. To illustrate the BLI benefit, a relationship between the amount of BLI and the net thrust benefit is established and analyzed for the electric fans at various fan pressure ratios. In addition, this relationship was also used to show the BLI benefit effect of using turbojet-like engines, which include pure turbojet engine and an engine with core only that drives separately attached multiple fans driven by mechanical, fluidic or electric means. Preliminary analysis indicates that the electric fan benefits most from the boundary layer ingestion. However, for the case of turbojet-like engines, this BLI benefit is very small or negative such that it is ideal to have them in free stream rather than embedding them within the airframe to take $100 \%$ total pressure recovery before the flow enters the inlet. Although the two processes are completely unrelated, the shock waves above a transonic airfoil also represent a loss of total pressure and velocity, much like the boundary layer flow. However, unlike the BLI-only propulsion system, the existence of shock waves above a transonic airfoil decreases the BLI benefit slightly. In real applications, it is possible to have both processes occurring in front of the BLI inlet for transonic vehicle.

\section{Acknowledgement}

The authors would like to thank Thomas Benson at NASA Glenn Research Center who provided helpful discussions and insights for the above analyses. In addition, a special thanks goes to NASA's Subsonic Fixed Wing (SFW) 
Project principal investigator, Ruben Delrosario, and project scientist, Richard Wahls, for their support on TeDP research activity.

\section{References}

${ }^{1}$ Kim, H.D., Berton, J.J., and Jones, S.M., "Low Noise Cruise Efficient Short Take-Off and Landing Transport Vehicle Study,” AIAA-2006-7738, Sep., 2006.

${ }^{2}$ Luongo C et al., "Next Generation More-Electric Aircraft: A Potential Application for HTS Superconductor,” Applied Superconductivity Conference Plenary Presentation, Paper 2AP01, August 17-22, 2008.

${ }^{3}$ Felder, J.L., Kim, H.D., and Brown, G.V., “Turboelectric Distributed Propulsion Engine Cycle Analysis for Hybrid-WingBody Aircraft,” AIAA-2009-1132, Jan. 2009.

${ }^{4}$ Smith, L.H. Jr., “Wake Ingestion Propulsion Benefit,” Journal of Propulsion and Power, Vol.9. No.1, Jan.-Feb. 1993.

${ }^{5}$ Plas, A.P., et al., "Performance of a Boundary layer Ingesting (BLI) Propulsion System,” AIAA-2007-450., Jan. 2007.

${ }^{6}$ Florea, R.V., et al., "Preliminary Design for Embedded Engine Systems," AIAA-2009-1131, Jan. 2009

${ }^{7}$ Drela, Mark, “Power Balance in Aerodynamic Flows,” AIAA-2009-3762, June, 2009. 